\title{
Diazoxide Attenuates Postresuscitation Brain Injury in a Rat Model of Asphyxial Cardiac Arrest by Opening Mitochondrial ATP-Sensitive Potassium Channels
}

\author{
Haidong Wu, ${ }^{1,2}$ Peng Wang, ${ }^{1,2}$ Yi Li, ${ }^{1,2}$ Manhui Wu, ${ }^{1,2}$ Jiali Lin, ${ }^{1,2}$ and Zitong Huang ${ }^{1,2}$ \\ ${ }^{1}$ Department of Emergency Medicine, Sun Yat-sen Memorial Hospital, Sun Yat-sen University, Guangzhou, China \\ ${ }^{2}$ Institute of Cardiopulmonary Cerebral Resuscitation, Sun Yat-sen University, Guangzhou, China
}

Correspondence should be addressed to Zitong Huang; syxhzt@163.com

Received 9 March 2016; Accepted 3 August 2016

Academic Editor: Konstantinos Pappas

Copyright (C) 2016 Haidong Wu et al. This is an open access article distributed under the Creative Commons Attribution License, which permits unrestricted use, distribution, and reproduction in any medium, provided the original work is properly cited.

\begin{abstract}
Objective. We investigated whether and how diazoxide can attenuate brain injury after cardiopulmonary resuscitation (CPR) by selective opening of mitochondrial ATP-sensitive potassium (mitoKATP) channels. Methods. Adult male Sprague-Dawley rats with induced cerebral ischemia ( $n=10$ per group) received an intraperitoneal injection of $0.1 \%$ dimethyl sulfoxide ( $1 \mathrm{~mL}$; vehicle group), diazoxide (10 mg/kg; DZ group), or diazoxide $(10 \mathrm{mg} / \mathrm{kg}$ ) plus 5-hydroxydecanoate $(5 \mathrm{mg} / \mathrm{kg}$; DZ + 5-HD group) $30 \mathrm{~min}$ after CPR. The control group (sham group, $n=5$ ) underwent sham operation, without cardiac arrest. Mitochondrial respiratory control rate (RCR) was determined. Brain cell apoptosis was assessed using TUNEL staining. Expression of Bcl-2, Bax, and protein kinase C epsilon (PKCE) in the cerebral cortex was determined by Western blotting and immunohistochemistry. Results. The neurological deficit scores (NDS) in the vehicle group decreased significantly at $24 \mathrm{~h}$ and $48 \mathrm{~h}$ after CPR. Diazoxide significantly improved NDS and mitochondrial RCR after CPR at both time points; 5-HD cotreatment abolished these effects. Diazoxide decreased TUNELpositive cells following $\mathrm{CPR}$, upregulated $\mathrm{Bcl}-2$ and $\mathrm{PKC} \varepsilon$, downregulated $\mathrm{Bax}$, and increased the $\mathrm{Bcl}-2 / \mathrm{Bax}$ ratio; 5-HD cotreatment reversed these effects. Conclusions. Diazoxide attenuates postresuscitation brain injury, protects mitochondrial function, inhibits brain cell apoptosis, and activates the PKC pathway by opening mitoKATP channels.
\end{abstract}

\section{Introduction}

Brain injury is the major cause of disability and death after cardiac arrest and CPR. Ischemia-reperfusion injury (IRI) after cardiac arrest and CPR leads to brain injury, resulting in cerebral cell necrosis, apoptosis, and oxidative stress injury. The brain is particularly vulnerable to IRI due to the specific structural and functional characteristics of brain tissue.

Although therapeutic hypothermia has been recommended for comatose survivors to improve neurologic outcomes following cardiac arrest, the efficacy of this treatment remains controversial, especially in children $[1,2]$. Therefore, alternative approaches are required to further improve neurologic outcomes in patients following cardiac arrest.

Following cardiac arrest and CPR, mitochondria play a critical role in producing and eliminating free radicals and in mediating oxidative stress injury. The pathophysiological mechanisms of brain damage after CPR are related to secondary mitochondrial injury and apoptosis; thus, therapeutic strategies aimed at preventing IRI-induced mitochondrial injury and apoptosis may improve functional recovery of the brain [3].

Ischemic preconditioning and postconditioning protect cells against IRI [4]. The mitochondrial ATP-sensitive potassium (mitoKATP) channel is important for protecting cells and organs from injury during ischemic preconditioning and postconditioning [5]. Recent evidence has suggested that the selective mitoKATP channel agonist diazoxide potently reduces mitochondrial injury by preserving mitochondrial integrity and inhibiting apoptosis in neurons $[6,7]$. Thus, targeting the mitoKATP channel may represent a novel approach to therapeutic strategies for neuroprotection [8].

Considering the serious consequences and poor prognosis of brain injury after cardiac arrest and CPR, we 
investigated the effects of diazoxide-mediated opening of mitoKATP channels on postresuscitation brain injury and aimed to elucidate the neuroprotective mechanisms of diazoxide in a rat model of cardiac arrest.

\section{Methods}

The study protocol was approved by the Sun Yat-sen University Animal Experimentation Ethics Committee (number SCXK2013-07-0028) and met the Guidelines for Ethical Conduct in the Care and Use of Experimental Animals published by the Ministry of Science and Technology of the People's Republic of China.

2.1. Animal Preparation. Male Sprague-Dawley rats (Experimental Animal Center of Sun Yat-Sen University, Grade II, Certification number SCXK (YUE) 2013-0029) weighing 300-400 g were fasted overnight, with free access to water. Global cerebral ischemia and reperfusion injuries were induced by asphyxial cardiac arrest followed by CPR. Rats were anesthetized by intraperitoneal injection of pentobarbital sodium ( $45 \mathrm{mg} / \mathrm{kg}$ body weight; Sigma, St. Louis, MO), with additional doses $(10 \mathrm{mg} / \mathrm{kg})$ added at intervals of approximately $1 \mathrm{~h}$, as necessary. Animals were orally intubated with a 14-gauge cannula (Abbocath-T, Hospira, Lake Forest, IL) into the trachea for ventilation.

We advanced a 23-gauge polyethylene 50 (PE-50) catheter (Abbocath-T) into the right external jugular vein for intravenous infusion and another into the thoracic aorta from the left femoral artery for measurement of mean arterial pressure (MAP). MAP was measured with a pressure transducer (BD, Franklin Lakes, NJ). Before insertion, the catheters were filled with physiological salt solution containing $5 \mathrm{IU} / \mathrm{mL}$ heparin to prevent blood coagulation. We maintained the rats' rectal temperature at $36.5 \pm 0.5^{\circ} \mathrm{C}$ using a heating lamp. Hemodynamic data and electrocardiograms were monitored with a multifunctional physiological monitor throughout the procedure (Spacelab Healthcare, Snoqualmie, WA).

2.2. Experimental Protocol. After baseline measurements were obtained, anesthetized rats were paralyzed with vecuronium bromide $2 \mathrm{mg} / \mathrm{kg}$ (Xianjupharmacy, Zhejiang, China). Asphyxia and cardiac arrest were induced by clamping the endotracheal tube according to the method described in our previous report [9]. Approximately 4 minutes following induction of apnea, cardiac arrest was determined by loss of aortic pulse and MAP $\leq 20 \mathrm{mmHg}$. Six minutes after the onset of cardiac arrest, precordial compression was initiated with an electrically driven mechanical chest compressor, while mechanical ventilation was initiated with pure oxygen $\left(\mathrm{FiO}_{2} 100 \%\right)$ at a tidal volume of $6 \mathrm{~mL} / \mathrm{kg}$ and a frequency of $50 \mathrm{breaths} / \mathrm{min}$. The precordial compression rate was maintained at 250/min and was synchronized with a compression/ventilation ratio of $5: 1$, with equal compressionrelaxation duration. We maintained arterial diastolic pressure at $25 \pm 5 \mathrm{mmHg}$ by adjusting the depth of compression. A 4-J biphasic waveform electrical shock (M-Series, Zoll Medical Corporation; Chelmsford, MA) was used to stop ventricular fibrillation (VF) if VF occurred after $4 \mathrm{~min}$ of
CPR. Adrenaline $(20 \mu \mathrm{g} / \mathrm{kg})$ was intravenously injected after 2 min of CPR. Return of spontaneous circulation (ROSC) was defined as return of a supraventricular rhythm with MAP $>60 \mathrm{mmHg}$, lasting for approximately $5 \mathrm{~min}$. The animals underwent intensive care with mechanical ventilation for $1 \mathrm{~h}$ after ROSC. Animals were extubated and placed in their cages after their upper airway reflexes became active.

2.3. Experimental Design. Thirty minutes after ROSC, rats were randomized into the vehicle-treated (vehicle), diazoxide-treated (DZ), or diazoxide +5 -hydroxydecanoate(5-HD-) treated (DZ + 5-HD) groups ( $n=10$ each). Vehicle group animals received $1 \mathrm{~mL}$ of $0.1 \%$ dimethyl sulfoxide (DMSO) intraperitoneally; DZ group animals received $10 \mathrm{mg} / \mathrm{kg}$ diazoxide intraperitoneally, while $\mathrm{DZ}+5-\mathrm{HD}$ group animals received $10 \mathrm{mg} / \mathrm{kg}$ diazoxide plus $5 \mathrm{mg} / \mathrm{kg}$ 5-HD intraperitoneally. Diazoxide (Sigma-Aldrich) was dissolved in $0.1 \%$ DMSO for injection, while 5-HD (SigmaAldrich) was dissolved in saline. A normal control group (sham group, $n=5$ ) also underwent identical anesthetic and surgical procedures without the induction of cardiac arrest.

2.4. Neurological Functional Testing. Functional neurological testing of the rats was performed $24 \mathrm{~h}$ and $48 \mathrm{~h}$ after ROSC by an observer blinded to experimental conditions. Neurological deficit scores (NDS) were digitalized on a scale of $0-80$ [10], based on a composite of arousal, reflex, motor, sensory, and balance responses, with 0 corresponding to brain death and 80 to no deficit.

2.5. Detection of Mitochondrial RCR. Half of the surviving animals in all groups were sacrificed by carbon dioxide asphyxiation $24 \mathrm{~h}$ after ROSC, and their brain tissues were removed for subsequent examinations. After rats were sacrificed, cortical tissues were rapidly separated, weighed, and placed in an ice-cold Dounce homogenizer. We isolated brain mitochondria as previously reported [11]. A Clark oxygen electrode system (OxygraphTM, Hansatech Instruments, King's Lynn, UK) was used to determine mitochondrial respiratory function. Reaction buffer $(2.5 \mathrm{~mL}$, consisting of $125 \mathrm{mM}$ potassium chloride, $2.5 \mathrm{mM} \mathrm{K \textrm {KH } _ { 2 }} \mathrm{PO}_{4}, 20 \mathrm{mM}$ HEPES, $4 \mathrm{mM}$ magnesium chloride, 0.1\% BSA, and $225 \mathrm{mM}$ mannitol) was stirred to a steady state in a sealed reaction tank at $25^{\circ} \mathrm{C}, \mathrm{pH}$ 7.4. After stabilization of the recorded curve, $2.5 \mathrm{~mL}$ of the mitochondrial suspension was added, and samples were incubated for $1 \mathrm{~min}$. We then added $20 \mu \mathrm{L}$ disodium succinate $(4 \mathrm{mM})$ to the tank, following which the oxygen concentration slowly declined, reflecting respiratory state 4 (R4). Next, we added $20 \mu \mathrm{L}$ of adenosine diphosphate (ADP, $50 \mathrm{mM}$ ), after which the oxygen concentration rapidly declined, reflecting respiratory state 3 (R3). The R3/R4 ratio reflected the respiratory control rate (RCR), which is a measure of the integrity of the mitochondrial membrane and oxidative phosphorylation: decreased RCR suggests impaired mitochondrial function.

2.6. TUNEL Staining. In order to quantify the rate of apoptosis, a terminal deoxynucleotidyl transferase-mediated dUTP 
nick-end labeling (TUNEL) assay was performed on paraffinembedded tissue sections of each animal. We performed TUNEL staining according to the manufacturer's instructions (Roche Molecular Biochemical, Mannheim, Germany) in order to identify apoptotic cells in paraffin sections of rat brains. We counted cells with nuclear brown-stained particles as TUNEL-positive in five randomly selected high-power fields (400x) on each slide. The apoptosis index (AI) in each group was averaged from the values determined using the following formula: $\mathrm{AI}=$ (number of apoptotic cells/total number of cerebral cells) $\times 100 \%$.

2.7. Western Blotting Analysis. Expression of Bcl-2, Bax, and $\mathrm{PKC} \varepsilon$ protein in the rat cortex was detected using Western blotting. The frozen tissue samples were completely homogenized in RIPA lysis buffer (Sigma-Aldrich) containing protease inhibitors. Lysates were clarified by centrifugation at $(10,800 \times \mathrm{g})$ for $15 \mathrm{~min}$ at $4^{\circ} \mathrm{C}$, and the lysate proteins were separated by sodium dodecyl sulfate polyacrylamide gel electrophoresis. We transferred the proteins to polyvinylidene fluoride membranes (Millipore, Billerica, MA) and blocked the membranes overnight $(5 \%$ milk powder in Tris-buffered saline). The membranes were incubated with mouse antiPKCe primary antibody (Abcam, Cambridge, MA), mouse anti-Bcl-2 primary antibody (Abcam), or mouse anti-Bax primary antibody (Abcam). Bands were visualized using the ECL Western Blotting Substrate Kit (Pierce, Rockford, IL). After scanning the blots, the intensity of the bands was determined using Image J version 7.0 (National Institutes of Health, Bethesda, MD) densitometry software. The resulting values were normalized against Glyceraldehyde 3-phosphate dehydrogenase (GAPDH) expression as an internal control. We performed a minimum of 3 blots for each protein analysis.

2.8. Immunohistochemistry Analysis. For immunohistochemistry, we deparaffinized the sections, which were subsequently washed three times in phosphate-buffered saline (PBS) for $5 \mathrm{~min}$. The sections were then blocked with $5 \%$ serum for $30 \mathrm{~min}$. Slides were incubated overnight with primary antibodies against $\mathrm{PKC} \varepsilon, \mathrm{Bcl}-2$, or Bax (Abcam) at $4^{\circ} \mathrm{C}$. After rinsing three times with PBS, slides were incubated with secondary antibodies at $37^{\circ} \mathrm{C}$ for $20-30 \mathrm{~min}$, followed by incubation with 3,30-diaminobenzidine tetrahydrochloride solution and counterstaining with hematoxylin. Five different high-power fields per slide were observed by light microscopy.

2.9. Statistical Analysis. All data are presented as mean \pm standard deviation (SD). Statistical power analyses were performed using SPSS version 19.0 (IBM Analytics, Chicago, IL, United States) for Windows. Unpaired $t$-tests were used to compare the parameters between two groups, while the Newman-Keuls test was used for multiple group comparisons. $P<0.05$ was considered statistically significant.

\section{Results}

3.1. Diazoxide Improves Neurological Outcomes. All shamoperated animals exhibited normal NDS at both time points

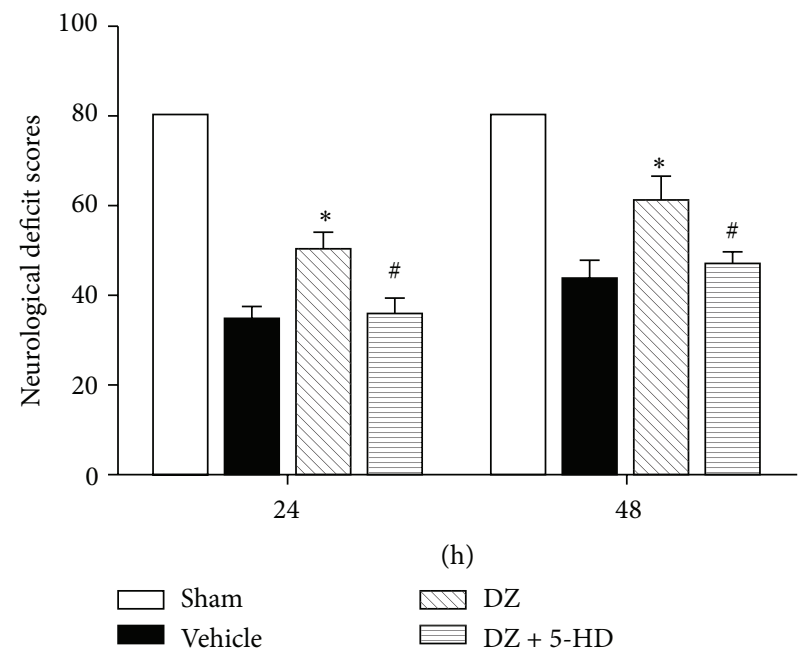

Figure 1: Neurological deficit scores for rats in the sham, vehicle group, DZ group, and DZ + 5-HD group at $24 \mathrm{~h}$ and $48 \mathrm{~h}$ after ROSC. Data are presented as means $\pm \mathrm{SD}, n=5$ rats/group. ${ }^{*} P<$ 0.05 versus vehicle group; ${ }^{\#} P<0.05$ versus DZ group. 5 -HD: 5 hydroxydecanoate; DZ: diazoxide; ROSC: return of spontaneous circulation.

(NDS: 80); however, NDS in the vehicle group decreased significantly at $24 \mathrm{~h}$ and $48 \mathrm{~h}$ after CPR. NDS improved significantly at $24 \mathrm{~h}$ and $48 \mathrm{~h}$ after CPR following diazoxide treatment, though this effect was abolished by cotreatment with 5-HD (Figure 1).

3.2. Diazoxide Improved Mitochondrial RCR. Mitochondrial RCR reflects the efficiency of oxidative phosphorylation as well as mitochondrial function. R3 and mitochondrial RCR of the vehicle group were significantly lower than those of the sham group $(P<0.05)$, while R3 and mitochondrial RCR of the DZ group were significantly higher than those of both the vehicle group and the $\mathrm{DZ}+5$ - HD group $(P<0.05) 24 \mathrm{~h}$ after ROSC (see Supplementary Figure 1 in Supplementary Material available online at http://dx.doi.org/ $10.1155 / 2016 / 1253842$ ). No significant differences in R4 were observed among the four groups at $24 \mathrm{~h}$ after ROSC. These results suggest that diazoxide protects mitochondrial respiratory function in rat brain cells after ROSC, but this effect can be abolished by cotreatment with 5-HD.

3.3. Diazoxide Inhibits Apoptosis in the Brain after CPR. TUNEL-positive cells in the cortical regions were scored as apoptotic. In the sham group, the percentage of TUNELpositive cells was markedly low (see Supplementary Figure 2). In the vehicle group; however, the percentage of TUNELpositive cells increased following CPR when compared to that of the sham group $(P<0.05)$. In the DZ group, the percentage of TUNEL-positive cells decreased significantly following CPR when compared to that of the vehicle group $(P<0.05)$. This effect was abolished by cotreatment with $5-$ $\mathrm{HD}$ (DZ versus DZ + 5-HD groups, $P<0.05$ ).

3.4. Diazoxide Increases the Bcl-2/Bax Ratio. Bcl-2 protein expression decreased significantly in the vehicle group 
following ROSC when compared to that of the sham group. Following administration of diazoxide, Bcl-2 protein expression increased significantly after ROSC when compared with that of the vehicle group, though this effect was negated by cotreatment with 5-HD. In contrast, Bax protein expression increased significantly following ROSC in the vehicle group when compared to that of the sham group. Compared to the vehicle group, Bax protein expression decreased significantly after administration of diazoxide in the DZ group; again, this effect was reversed upon cotreatment with 5-HD. Changes in the $\mathrm{Bcl}-2 / \mathrm{Bax}$ ratio also remained consistent with these variations (see Supplementary Figures 2 and 3).

3.5. Diazoxide Increases PKCE Expression. PKCE protein expression decreased significantly following ROSC in the vehicle group when compared to that of the sham group. After administration of diazoxide, $\mathrm{PKC} \varepsilon$ protein expression increased significantly following ROSC when compared to that of the vehicle group. This effect was again abolished by cotreatment with 5-HD (see Supplementary Figure 4).

\section{Discussion}

The results of the present study indicate that administration of diazoxide following global cerebral ischemia/reperfusion induced by cardiac arrest/CPR may protect mitochondrial respiration and energy synthesis, inhibit apoptosis, and improve neurological function by opening mitoKATP channels. These results are consistent with those of previous studies that have reported the neuroprotective effects of diazoxide $[7,8]$.

Diazoxide preconditioning has been proven effective for the treatment of focal brain injuries [12-14]; however, preconditioning is infeasible for global brain injuries following unpredictable experiences of cardiac arrest. A previous study has observed that diazoxide postconditioning can attenuate ischemia/reperfusion-induced injuries in the rat liver [15] and induce mitochondrial protein Snitrosylation as well as redox-sensitive mitochondrial phosphorylation/translocation of RISK elements [16]. The results of the present study also demonstrate that diazoxide postconditioning may be a potential therapeutic strategy for the treatment of global brain injury following cardiac arrest.

The pathophysiologic mechanisms of brain damage secondary to cardiac arrest and CPR include temporary global cerebral ischemia and reperfusion injury, impairment of mitochondrial energy functions, and secondary apoptosis during and after CPR [17]. Some studies have reported that postischemic inflammatory responses and apoptosis are involved in brain damage after cerebral ischemia [18]. Interventions that can reduce secondary brain injury may improve survival and neurological recovery, though most brain injuries cannot be rapidly treated following cardiac arrest and CPR, and the cascade of inflammatory responses and apoptosis may be inevitable; effective measures that can prevent brain injury after CPR remain limited.

Inhibition of postresuscitation mitochondrial apoptotic executioners may also provide considerable neuroprotection [3]. Some studies have shown that opening of the mitoKATP channel causes mitochondrial membrane depolarization, decreased mitochondrial membrane potential, and increased mitochondrial volume and that this has antiapoptotic effects, resulting in PKC activation and inhibition of reactive oxygen species (ROS) production during ischemia [19]. When taken with these previous findings, the results of the present study indicate that diazoxide, which opens mitoKATP channels, may attenuate cerebral injuries induced by hypoperfusion or ischemia/reperfusion $[6,7]$.

Mitochondria, the energy-producing power stations of the cell, play a critical role as effectors and targets of ischemia/reperfusion injury following cardiac arrest. Mitochondria are involved in oxidative stress, calcium overload, and apoptosis cascades that occur during IRI after CPR. Previously, our group [20] and others [21] have demonstrated that myocardial mitochondrial function is impaired and that mitochondrial ultrastructure changes occur during cardiac arrest and following ROSC. Further research has indicated that cerebral mitochondrial oxidative phosphorylation is also impaired following CPR in a rat model of ventricular fibrillation [11]. Xu et al. observed that RCR decreases after resuscitation in a rat model of $\mathrm{KCl}$-induced cardiac arrest [22]. However, little is known regarding the dysfunction of cerebral mitochondrial during cardiac arrest and following ROSC. In the present study, we demonstrated that mitochondrial R3 and RCR decreased significantly following ROSC and that this decrease is significantly attenuated by diazoxide. Furthermore, the effect of diazoxide was abolished by 5-HD, a specific antagonist of the mitoKATP channel. Therefore, diazoxide may be a potential drug for improving cerebral mitochondrial function after CPR via opening of mitoKATP channels.

Apoptosis after cardiac arrest is another important cause of neuronal degeneration, leading to neurological dysfunction after global cerebral ischemia [23]. Interactions between the proapoptotic and antiapoptotic proteins of the $\mathrm{Bcl}-2$ family on the outer mitochondrial membrane are important in apoptosis. Upregulation of Bcl-2 or downregulation of Bax has been found to attenuate apoptosis; hence, an increased $\mathrm{Bcl}-2 / \mathrm{Bax}$ protein ratio inhibits neuronal apoptosis [3]. However, effective approaches for preventing or limiting neuronal damage due to apoptosis after cardiac arrest remain elusive. Recently, researchers have observed that diazoxide attenuates graft injury and suppresses hepatic ischemia/reperfusion injury after mouse liver transplantation via a Bcl-2-dependent mechanism [24]. In the present study, we revealed that diazoxide enhances $\mathrm{Bcl}-2$ expression and inhibits Bax expression, thereby increasing the Bcl-2/Bax ratio and inhibiting apoptosis following CPR. Altogether, these results suggest that diazoxide may suppress apoptosis that occurs in conjunction with postresuscitation global cerebral ischemia and may contribute to neurological functional recovery by opening mitoKATP channels.

PKC regulates key cytoprotective mitochondrial functions, including electron transport chain activity, ROS generation, mitochondrial permeability transition, and detoxification of reactive aldehydes [25]. Activation of PKC also protects mitochondria by activating mitoKATP channels [26], while downregulation of PKC inhibits diazoxideinduced activation of mitoKATP channels [27]. Activation of 
mitoKATP channels for cardiac protection against ischemic injury is dependent on PKC activity [28]. PKC $\varepsilon$ is an isoform of $\mathrm{PKC}$ that regulates mitochondrial pools of nicotinamide adenine dinucleotide (NAD) or nicotinamide phosphoribosyl transferase and affects ischemic preconditioning [29]. In a study of myocytes, researchers observed that opening of mitoKATP channels may activate PKC $\varepsilon$ and induce its translocation into myofibrillar-like structures. PKC $\varepsilon$ activation occurs downstream of the mitoKATP channel, possibly as a result of ROS production after the opening of mitoKATP channels [30]. In the present study, we demonstrated that diazoxide can increase $\mathrm{PKC} \varepsilon$ expression but that this increase in expression is abrogated by 5 -HD. The neuroprotective effect of diazoxide after CPR may occur via activation of the PKC pathway after opening of mitoKATP channels.

The present study possesses some limitations. As this is a rat model, care should be exercised when extrapolating the results to human patients. Further research utilizing animal models more similar to humans with respect to cardiac arrest and CPR is required prior to clinical studies of the neuroprotective effects of diazoxide following CPR.

\section{Conclusion}

In conclusion, the results of the present study indicate that diazoxide-induced opening of mitoKATP channels attenuates postresuscitation brain injury and protects against impaired mitochondrial function, modulates the expression of apoptotic proteins, inhibits cell apoptosis, and activates the protective PKC signaling pathway. Our findings provide new insight into strategies for improving the therapeutic efficacy of treatments for postresuscitation brain injury. However, the precise mechanisms underlying the opening of mitoKATP channels and neuroprotective activity of PKC remain to be elucidated.

\section{Competing Interests}

The authors declare that there are no competing interests regarding the publication of this paper.

\section{Acknowledgments}

This study was supported by two grants from the Science and Technology Plan Project of Guangdong Province (nos. 2012B031800084 and 2014A020212624).

\section{References}

[1] K. S. Bae, S. D. Shin, Y. S. Ro et al., "The effect of mild therapeutic hypothermia on good neurological recovery after outof-hospital cardiac arrest according to location of return of spontaneous circulation: a nationwide observational study," Resuscitation, vol. 89, pp. 129-136, 2015.

[2] J. F. Bistritz, L. M. Horton, and A. Smaldone, "Therapeutic hypothermia in children after cardiac arrest: a systematic review and meta-analysis," Pediatric Emergency Care, vol. 31, no. 4, pp. 296-303, 2015.

[3] L. Galluzzi, E. Morselli, O. Kepp, and G. Kroemer, "Targeting post-mitochondrial effectors of apoptosis for neuroprotection,"
Biochimica et Biophysica Acta-Bioenergetics, vol. 1787, no. 5, pp. 402-413, 2009.

[4] A. A. Galle and N. M. Jones, "The neuroprotective actions of hypoxic preconditioning and postconditioning in a neonatal rat model of hypoxic-ischemic brain injury," Brain Research, vol. 1498, pp. 1-8, 2013.

[5] Y. Nakai, H. Horimoto, S. Mieno, and S. Sasaki, "Mitochondrial ATP-sensitive potassium channel plays a dominant role in ischemic preconditioning of rabbit heart," European Surgical Research, vol. 33, no. 2, pp. 57-63, 2001.

[6] Y. Zhu, C. C. Wendler, O. Shi, and S. A. Rivkees, "Diazoxide promotes oligodendrocyte differentiation in neonatal brain in normoxia and chronic sublethal hypoxia," Brain Research, vol. 1586, pp. 64-72, 2014.

[7] X. He, X. Mo, H. Gu et al., "Neuroprotective effect of diazoxide on brain injury induced by cerebral ischemia/reperfusion during deep hypothermia," Journal of the Neurological Sciences, vol. 268, no. 1-2, pp. 18-27, 2008.

[8] I. Nakagawa, D. Wajima, K. Tamura, F. Nishimura, Y.-S. Park, and H. Nakase, "The neuroprotective effect of diazoxide is mediated by mitochondrial ATP-dependent potassium channels in a rat model of acute subdural hematoma," Journal of Clinical Neuroscience, vol. 20, no. 1, pp. 144-147, 2013.

[9] Q.-M. Lin, S. Zhao, L.-L. Zhou, X.-S. Fang, Y. Fu, and Z.-T. Huang, "Mesenchymal stem cells transplantation suppresses inflammatory responses in global cerebral ischemia: contribution of TNF- $\alpha$-induced protein 6," Acta Pharmacologica Sinica, vol. 34, no. 6, pp. 784-792, 2013.

[10] R. G. Geocadin, R. Ghodadra, T. Kimura et al., "A novel quantitative EEG injury measure of global cerebral ischemia," Clinical Neurophysiology, vol. 111, no. 10, pp. 1779-1787, 2000.

[11] J. Jiang, X. Fang, Y. Fu, W. Xu, L. Jiang, and Z. Huang, "Impaired cerebral mitochondrial oxidative phosphorylation function in a rat model of ventricular fibrillation and cardiopulmonary resuscitation," BioMed Research International, vol. 2014, Article ID 192769, 9 pages, 2014.

[12] H. Dong, S. Wang, Z. Zhang, A. Yu, and Z. Liu, "The effect of mitochondrial calcium uniporter opener spermine on diazoxide against focal cerebral ischemia-reperfusion injury in rats," Journal of Stroke and Cerebrovascular Diseases, vol. 23, no. 2, pp. 303-309, 2014.

[13] L. Zhang, S. Wang, H. Dong et al., "Spermine attenuates the preconditioning of diazoxide against transient focal cerebral ischemia in rats," Neurological Research, vol. 36, no. 7, pp. 666-672, 2014.

[14] J. G. Shake, E. A. Peck, E. Marban et al., "Pharmacologically induced preconditioning with diazoxide: a novel approach to brain protection," Annals of Thoracic Surgery, vol. 72, no. 6, pp. 1849-1854, 2001.

[15] Y. S. Tian, T. Z. Rong, Y. L. Hong, L. Min, and P. G. Jian, "Pharmacological postconditioning with diazoxide attenuates ischemia/reperfusion-induced injury in rat liver," Experimental and Therapeutic Medicine, vol. 5, no. 4, pp. 1169-1173, 2013.

[16] C. Penna, M.-G. Perrelli, F. Tullio et al., "Diazoxide postconditioning induces mitochondrial protein S-Nitrosylation and a redox-sensitive mitochondrial phosphorylation/translocation of RISK elements: no role for SAFE," Basic Research in Cardiology, vol. 108, no. 5, article 371, 2013.

[17] S. T. Yeh, H.-L. Lee, S. E. Aune, C.-L. Chen, Y.-R. Chen, and M. G. Angelos, "Preservation of mitochondrial function with cardiopulmonary resuscitation in prolonged cardiac arrest in rats," 
Journal of Molecular and Cellular Cardiology, vol. 47, no. 6, pp. 789-797, 2009.

[18] Y. Yuan, Q. Guo, Z. Ye, X. Pingping, N. Wang, and Z. Song, "Ischemic postconditioning protects brain from ischemia/ reperfusion injury by attenuating endoplasmic reticulum stressinduced apoptosis through PI3K-Akt pathway," Brain Research, vol. 1367, pp. 85-93, 2011.

[19] D. W. Busija, Z. Lacza, N. Rajapakse et al., "Targeting mitochondrial ATP-sensitive potassium channels-a novel approach to neuroprotection," Brain Research Reviews, vol. 46, no. 3, pp. 282-294, 2004.

[20] X. Fang, Z. Huang, J. Zhu et al., "Ultrastructural evidence of mitochondrial abnormalities in postresuscitation myocardial dysfunction," Resuscitation, vol. 83, no. 3, pp. 386-394, 2012.

[21] F. Han, T. Da, N. A. Riobo, and L. B. Becker, "Early mitochondrial dysfunction in electron transfer activity and reactive oxygen species generation after cardiac arrest," Critical Care Medicine, vol. 36, no. 11, pp. S447-S453, 2008.

[22] K. Xu, M. A. Puchowicz, X. Sun, and J. C. LaManna, "Mitochondrial dysfunction in aging rat brain following transient global ischemia," Advances in Experimental Medicine and Biology, vol. 614, pp. 379-386, 2008.

[23] L. J. Martin, N. A. Al-Abdulla, A. M. Brambrink, J. R. Kiesch, F. E. Sieber, and C. Portera-Cailliau, "Neurodegeneration in excitotoxicity, global cerebral ischemia, and target deprivation: a perspective on the contributions of apoptosis and necrosis," Brain Research Bulletin, vol. 46, no. 4, pp. 281-309, 1998.

[24] Q. Wu, C. Tang, Y.-J. Zhang et al., "Diazoxide suppresses hepatic ischemia/reperfusion injury after mouse liver transplantation by a BCL-2-dependent mechanism," Journal of Surgical Research, vol. 169, no. 2, pp. e155-e166, 2011.

[25] X. Sun, G. R. Budas, L. Xu, G. E. Barreto, D. Mochly-Rosen, and R. G. Giffard, "Selective activation of protein kinase C in mitochondria is neuroprotective in vitro and reduces focal ischemic brain injury in mice," Journal of Neuroscience Research, vol. 91, no. 6, pp. 799-807, 2013.

[26] P. Korge, H. M. Honda, and J. N. Weiss, "Protection of cardiac mitochondria by diazoxide and protein kinase C: implications for ischemic preconditioning," Proceedings of the National Academy of Sciences of the United States of America, vol. 99, no. 5, pp. 3312-3317, 2002.

[27] Y. Wang, E. Takashi, M. Xu, A. Ayub, and M. Ashraf, "Downregulation of protein kinase $\mathrm{C}$ inhibits activation of mitochondrial $\mathrm{K}_{\text {ATP }}$ channels by diazoxide," Circulation, vol. 104, no. 1, pp. 8590, 2001.

[28] Y. Wang, K. Hirai, and M. Ashraf, "Activation of mitochondrial ATP-sensitive $\mathrm{K}^{+}$channel for cardiac protection against ischemic injury is dependent on protein kinase C activity," Circulation Research, vol. 85, no. 8, pp. 731-741, 1999.

[29] K. C. Morris-Blanco, C. H. Cohan, J. T. Neumann, T. J. Sick, and M. A. Perez-Pinzon, "Protein kinase $\mathrm{C}$ epsilon regulates mitochondrial pools of Nampt and NAD following resveratrol and ischemic preconditioning in the rat cortex," Journal of Cerebral Blood Flow and Metabolism, vol. 34, no. 6, pp. 10241032, 2014.

[30] H. Li, T. Yang, Z. Long, and J. Cheng, "Effect of mitochondrial ATP-sensitive potassium channel opening on the translocation of protein kinase C epsilon in adult rat ventricular myocytes," Genetics and Molecular Research, vol. 13, no. 2, pp. 4516-4522, 2014. 

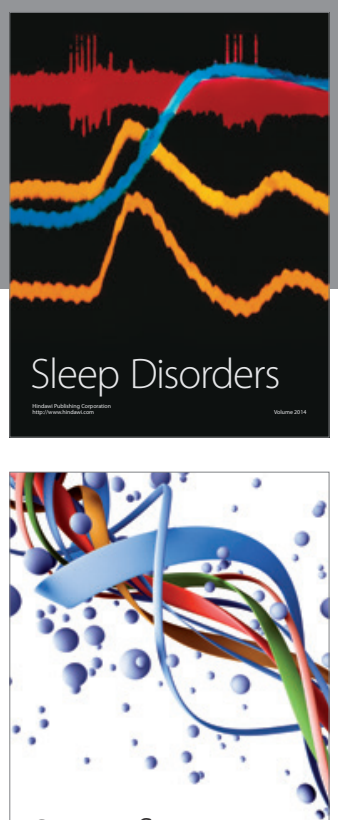

Scientifica
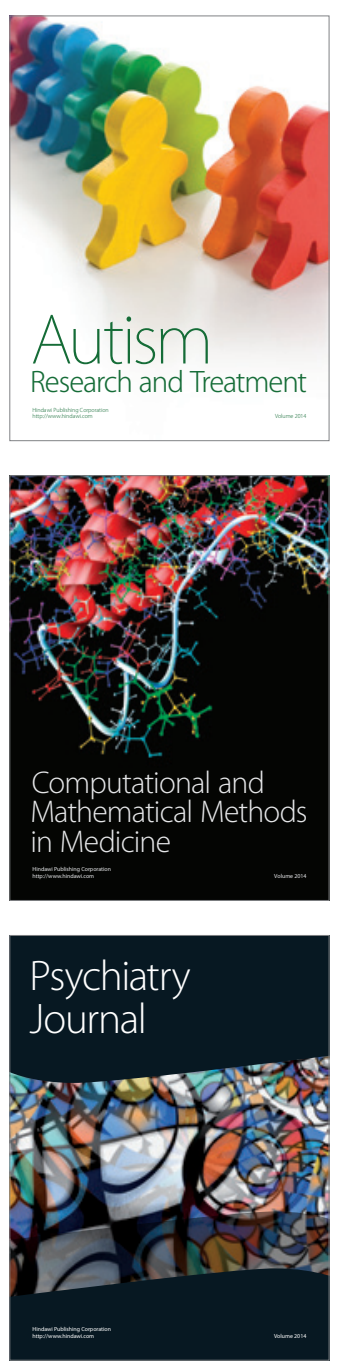
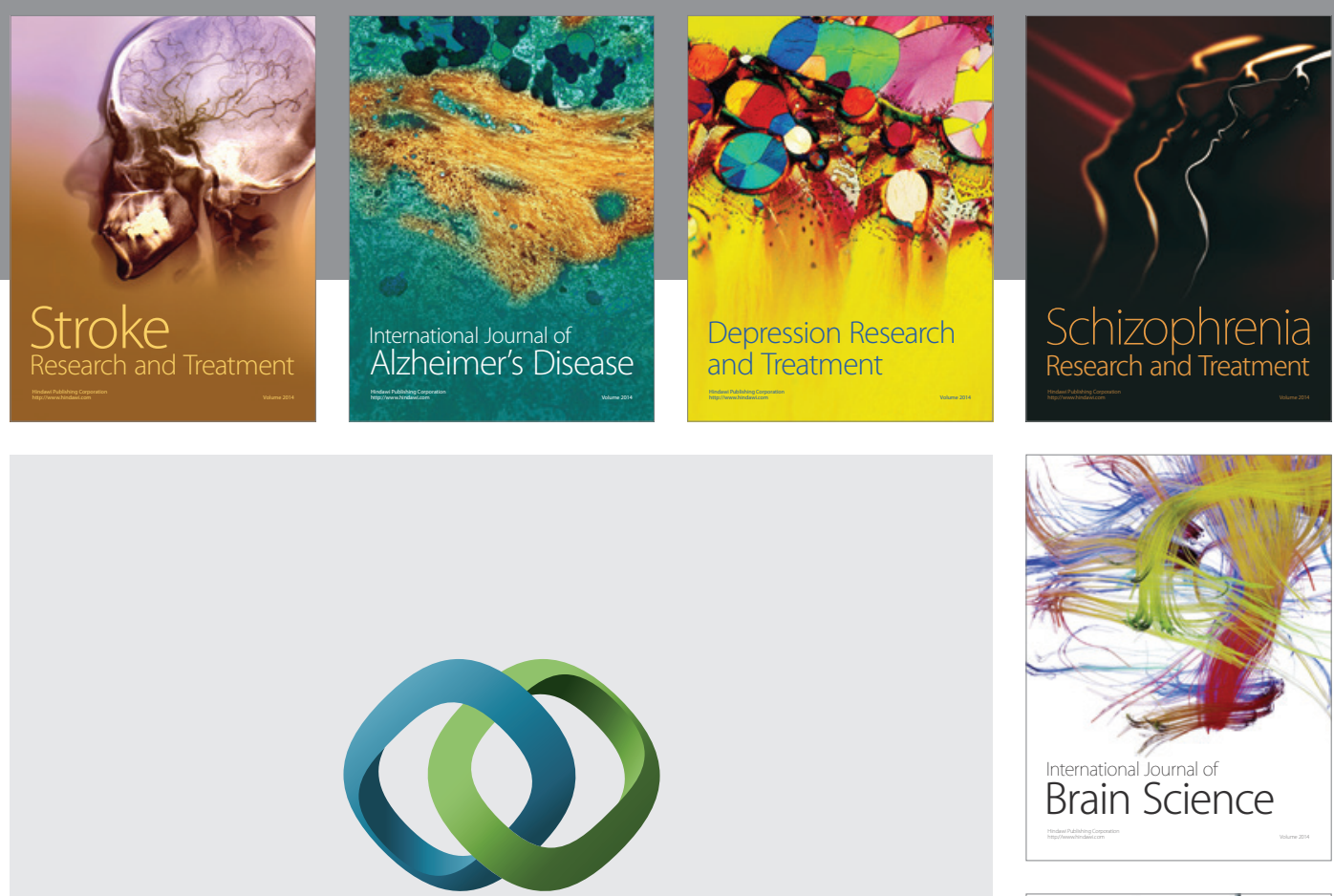

\section{Hindawi}

Submit your manuscripts at

http://www.hindawi.com
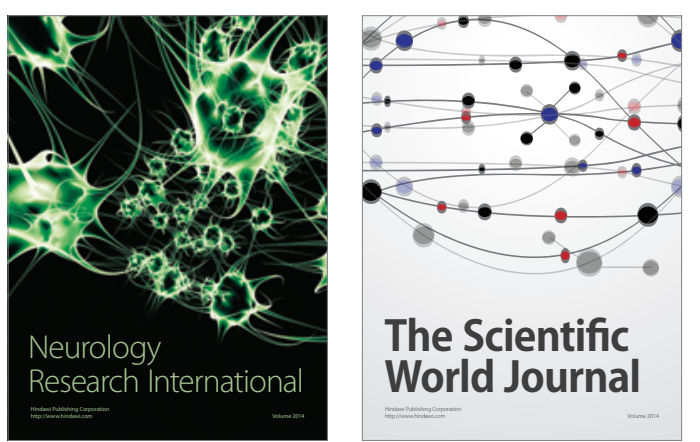

The Scientific World Journal

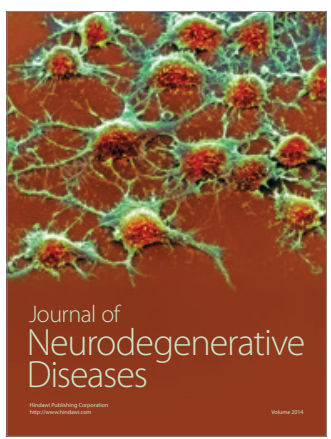

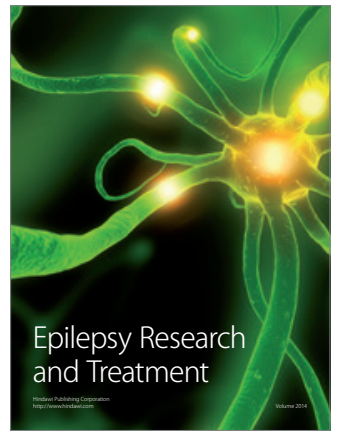

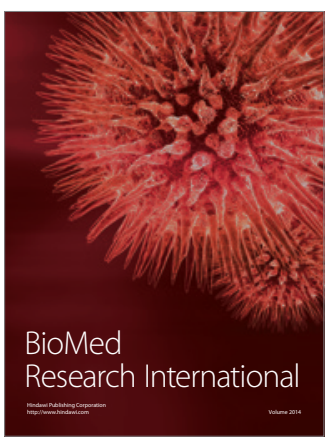

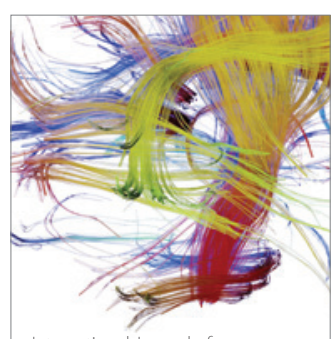

Brain Science

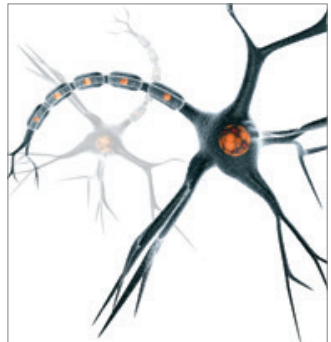

Neural Plasticity
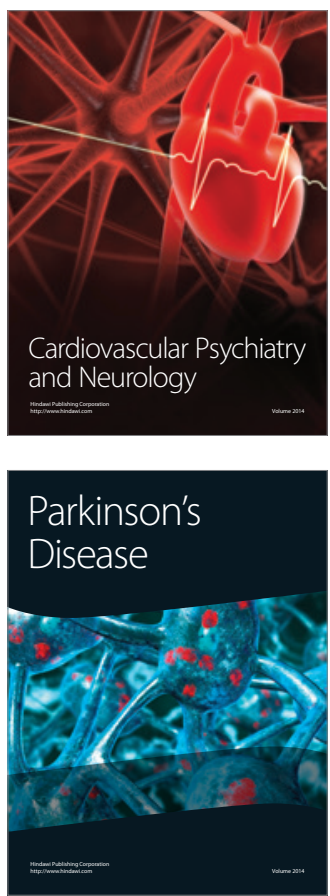